Pacific Journal of Mathematics

THE FOURIER TRANSFORM FOR NILPOTENT LOCALLY 


\title{
THE FOURIER TRANSFORM FOR NILPOTENT LOCALLY COMPACT GROUPS: I
}

\author{
ROGER E. HowE
}

\begin{abstract}
In his work on nilpotent lie groups, A. A. Kirillov introduced the idea of classifying the representations of such groups by matching them with orbits in the dual of the lie algebra under the coadjoint action. His methods have proved extremely fruitful, and subsequent authors have refined and extended them to the point where they provide highly satisfactory explanations of many aspects of the harmonic analysis of various lie groups. Meanwhile, it appears that nonlie groups are also amenable to such an approach. In this paper, we seek to indicate that, indeed, a very large class of separable, locally compact, nilpotent groups have a Kirillovtype theory.
\end{abstract}

On the other hand, elementary examples show that not all such groups can have a perfect Kirillov theory. The precise boundary between good and bad groups is not well defined, and varies with the amount of technical complication you can tolerate. At this stage, the delineation of the boundary is the less rewarding part of the theory, and will be deferred to a future publication. In the present paper, we lay some groundwork, and then discuss a particularly nice special case, which also has significance in the general picture.

Since Kirillov's approach hinges on the use of the lie algebra and its dual, the first concern in imitating his theory is to find a lie algebra. In $\S \mathrm{I}$, we consider the generalities of the algebraic aspects of this problem. We rely very heavily on Serre [10]. Indeed, the beginning of $\S I$ is a summary of the fourth chapter of [10], with differences in emphasis to fit the present need. The major tool is the Campbell-Hausdorff formula, which we use to prove some elementary facts on nilpotent groups, as well as to construct lie algebras.

In $\S I I$, we discuss the structure theory of locally compact nilpotent groups. We should emphasize here that we are dealing with groups which are genuinely nilpotent in the algebraic sense, and which have a topology. We do not consider groups which are nilpotent only in some topological sense. Specifically, $G$ is $k$-step nilpotent if the ascending central series $\mathscr{Z}(G)$, $\mathscr{Z}^{(2)}(G) \cdots$, satisfies $\mathscr{Z}^{(k)}(G)=G$. Alternatively, if $x, y \in G$, define the commutator $(x, y)$ of $x$ and $y$ by $(x, y)=x^{-1} y^{-1} x y$. Define the order of a commutator inductively: all $x \in G$ have order one; the commutator of commutators of orders $i$ 
and $j$ has order $i+j . \quad G^{(i)}$ is the closed subgroup generated by all $i$ th order commutators. $G$ is $k$-step nilpotent if $G^{(k+1)}=\{e\}$, the trivial subgroup.

Let us say a locally compact group $G$ is a quasi-torsion group if every $x \in G$ generates a subgroup whose closure is compact and pro-finite. $G$ is a quasi- $p$ group, if every $x \in G$ generates a pro- $p$ group. We will show that a locally compact nilpotent group $G$ is built in a fairly well defined way out of its identity component, a discrete group, and a quasi-torsion group. Since the identity component is very much like a lie group, and since at least finitely generated discrete groups have been analyzed in [5], we shall focus our attention in this paper on the harmonic analysis of quasi-torsion groups. Since we can show that a quasi-torsion nilpotent group is the restricted direct product of quasi- $p$ groups, in direct generalization of the classical Sylow decomposition of finite nilpotent groups, we need actually consider only quasi-p groups. For these groups, we establish, at the end of $\S I I$,

THEOREM I. Let $\mathscr{P}$ be a k-step nilpotent separable locally compact quasi-p group, with $p>k$. Then there is a locally compact abelian quasi-p group $P$, equipped with a bilinear operation [, ]: $P \times P \rightarrow P$, making into a k-step nilpotent locally compact lie algebra; and there is a homeomorphism exp: $P \rightarrow \mathscr{P}$, with inverse denoted by log, which sends Haar measure to Haar measure, and establishes a bijection between closed subgroups of $\mathscr{P}$ and closed subalgebras of $P$, and satisfies the Campbell-Hausdorff formula. Moreover, inner automorphisms of $\mathscr{P}$ pull back via exp to define an action Ad of $\mathscr{P}$ on $P$ by measure-preserving automorphisms. $P$ and exp are unique up to isomorphism.

This theorem forms the basis of our analysis of the representations of $\mathscr{P}$, carried out in $\S$ III. For any locally compact group $G$, we let $C^{*}(G)$ be the enveloping $C^{*}$-algebra of $L_{1}(G)$, and $M(G)$ the primitive ideal space of $C^{*}(G)$. Let $C(G)$ (with no star) be the continuous functions, $C_{0}(G)$ those with compact support. If $G$ is totally disconnected, we let $\mathscr{A}(G)$ stand for the space of locally constant functions of compact support-the Schwartz-Bruhat space. If $H \subseteq G$ is a closed subgroup, $V$ a representation of $H$, then $U^{V, H}$, or $U^{V}$, (or $U^{\psi, H}$, or $U^{\psi}$ if $V$ is finite dimensional with character $\psi$ ) denotes the representation of $G$ induced from $V$ on $H$. Recall that a representation $U$ of $G$ is CCR if $U\left(L_{1}(G)\right)$ consists of compact operators.

Returning now to $\mathscr{P}$, if $\mathscr{R} \subseteq \mathscr{P}$ is a subgroup, then $R=\log \mathscr{R}$ will denote its inverse image in $P$. Similarly, if $R \cong P$ is a subalgebra, then $\mathscr{R}=\exp R$ will be the corresponding subgroup of $\mathscr{P}$. Con- 
jugation in $\mathscr{P}$ will induce actions on various objects, and one of these actions will be denoted by Ad or $\mathrm{Ad}^{*}$, depending on whether it seems like a direct action or a dual action. In particular, we have an action $\mathrm{Ad}^{*}$ on $\hat{P}$, the Pontryagin dual of $P$, and we will denote the quasiorbit space, that is the space of orbit closures, of the action, by $Q(\hat{P})$. It has, of course, the quotient topology.

We will, whenever convenient, identify functions on $\mathscr{P}$ and $P$ by means of exp and log. In particular, we take $\mathscr{A}(\mathscr{P})$ into $\mathscr{A}(P)$, and then by means of the Fourier transform, to $\mathscr{A}(\hat{P})$. This will be referred to as the Fourier transform for $\mathscr{P}$.

Finally, if $R \subseteq P$ is a subgroup, and $\psi \in \hat{P}$, we will say $R$ is subordinate to $\psi$ if $\psi([R, R])=1$, or equivalently (in our case of $p>k$ ) if $\psi$ defines a one-dimensional character of $\mathscr{R}$, if $R$ is a subalgebra.

The main result of the paper summarizes for $\mathscr{P}$ the facts of harmonic analysis we have come to expect for nilpotent groups.

THEOREM II. There is a canonical homeomorphism $\alpha: Q(\hat{P}) \rightarrow$ $M(\mathscr{P})$. Given an $\mathrm{Ad}^{*} \mathscr{P}$ quasiorbit $\theta \subseteq \hat{P}$, and $\psi \in \theta$, then there are subalgebras $R \subseteq P$, subordinate to $\psi$, such that $U^{\psi, R}$ is irreducible, with kernel $\alpha(\theta)$. Such a representation is CCR if $\theta$ is actually an orbit; in which case the induced representation is of course independent of $\psi$ and $R$; and $\mathscr{A}(\mathscr{P})$ is sent to operators of trace class. The Fourier transform for $\mathscr{P}$ defines a bijection between Ad $\mathscr{P}$-invariant positive definite functionals on $\mathscr{A}(\mathscr{P})$ and $\mathrm{Ad}^{*} \mathscr{P}$ invariant positive measures on $\hat{P}$. For quasiorbits $\theta$ which are actually orbits, (and so homogeneous spaces), the unique $\mathrm{Ad}^{*} \mathscr{P}_{-}$ invariant measure supported by $\theta$ defines (a multiple of) the canonical trace. The Plancherel measure for $\mathscr{P}$ corresponds to Haar measure on $\hat{P}$.

We establish Theorem II as a sequence of propositions, rather than in one piece.

I. Nilpotent groups and lie algebras. Nilpotent groups are relatively easy to analyze because they form a fairly neat category, with manageable universal objects and close connections with abelian groups. Here we systematically expose the consequences of these facts, largely following [10].

Let $N$ be a $k$-step nilpotent group, with some set $X=\left\{X_{i}\right\}$ of generators. Let $F(X)$ be the free group on $X$. Then there is a natural homomorphism $\alpha: F(X) \rightarrow N$. Since $N$ is $k$-step nilpotent, $\alpha$ is trivial on $F^{(k+1)}$, and so factors to a map on $F / F^{(k+1)}=N_{k}(X)$, 
which we may refer to as the free $k$-step nilpotent group on $X$, since it clearly has the universal property suggested by that name.

There are natural surjective homomorphisms $\pi_{k}: N_{k}(X) \rightarrow N_{k-1}(X)$. The kernel of $\pi_{k}$ is $\mathscr{z}\left(N_{k}\right)=N_{k}^{(k)}$. (In general $\mathscr{\varkappa}^{j}\left(N_{k}\right)=N_{k}^{(k+1-j)}$.) $\mathscr{\digamma}\left(N_{k}\right)$ is known to be a free abelian group, and its rank is finite and known if ${ }^{*}(X)$, the cardinality of $X$, is finite. In particular $N_{k}(X)$ is torsion free.

Now, given a set $X$, and a commutative, associative ring with unit $A$, one may construct the free $A$-lie algebra over $X$ as follows. Take $M(X)$, the set of all nonassociative words in the elements of $X$, that is, the smallest set containing $X$ and containing all ordered pairs formable from elements of itself. $(M(X)$ is also known as the free magma on $X$.) $M(X)$ has a natural law of composition, i.e., taking ordered pairs. The free $A$-module on $M(X)$, with multiplication extended $A$-linearly, is the free $A$-algebra over $X$. It is not associative. Dividing out by the ideal generated by all elements of the forms $a a$ and $(a(b c))+(c(a b))+(b(c a))$ yields $\mathscr{L}(X, A)$, the free $A$-lie algebra on $X$. It has the appropriate universal property. It also has a natural graded structure, $\mathscr{L}=\bigoplus_{i=1}^{\infty} L_{i}$, derived from word length in $M(X) . \mathscr{L}^{(j)}$, the $j$ th group in the descending central series of $\mathscr{L}$, is $\bigoplus_{i=j}^{\infty} L_{i}$. Thus one may consider $\mathscr{L}_{k}=\mathscr{L} / \mathscr{L}^{(k+1)}\left(=\bigoplus_{i=1}^{k} L_{i}\right.$ as $A$-modules) to be the free $k$-step nilpotent $A$-lie algebra on $X$, again with evident universal property.

We may also construct from $X$, the tensor algebra $T(X, A)$ over $A(X)$, the free $A$-module over $X . T(X, A)$ is also the free associative algebra over $X$. The lie subalgebra of $T(X, A)$ generated by $X$ is isomorphic to $\mathscr{L}(X, A)$, and $T(X, A)$ is isomorphic to the universal enveloping algebra of $\mathscr{L}(X, A) . T$ is of course graded, and the gradings of $\mathscr{L}$ and $T$ are consistent, i.e., $L_{i}=\mathscr{L} \cap T_{i}$. If we consider $T / \bigoplus_{i=k+1}^{\infty} T_{i}=\mathscr{T}_{k}$, then $\mathscr{T}_{k}$ is an appropriately nilpotent enveloping algebra for $\mathscr{L}_{k}$.

Returning to $F(X)$, we consider $g r F(X)=\bigoplus_{i=1}^{\infty} F^{(i)} / F^{(i+1)}$. The commutator operation $(x, y)$ factors to a bilinear map on $\operatorname{gr} F(X)$, making this into a lie algebra, and with this structure $g r F$ is isomorphic to $\mathscr{L}(X, Z)$. At this point, it is appropriate to remark that for any $A, \mathscr{L}(X, A) \cong \mathscr{L}(X, Z) \otimes_{z} A$; and similarly for the various other universal objects we have mentioned.

Now suppose division by $k$ ! is permissible in $A$. Then we may define exp: $\mathscr{T}_{k} \rightarrow \mathscr{T}_{k}$ and its inverse, log, by the truncations of the usual formulas. $\mathscr{N}_{k}(X, A)$, the image of $\mathscr{L}_{k}(X, A)$ under exp, is a multiplicative group. If $A$ is torsion free, then $\left\{\exp X_{i}: X_{i} \in X\right\}$ generates a group isomorphic to $N_{k}(X)$, and the natural filtration of $N_{k}$ is consistent with the filtration given by the $\exp \mathscr{L}_{k}^{(j)}$. Since $\mathscr{N}_{k}(X, A)$ is a group, given $y_{1}, \cdots, y_{n} \in \mathscr{L}_{k}, \prod_{i=1}^{n} \exp y_{i}=\exp z$ for 
some $z \in \mathscr{L}_{k}$. The specific functional relation between the $y_{i}$ and $z$ is the Campbell-Hausdorff formula, which we will refer to as C. $\mathrm{H}$.

Before giving $\mathrm{C} . \mathrm{H}$. in an explicit form, we introduce some numbers, useful in using it. $\alpha(j)$ is defined as follows: let $p$ be a prime, and let $j=\Sigma c_{i} p^{i}$, with $0 \leqq c_{i}<p$; put $r_{p}(j)=\Sigma c_{i}$, and $\alpha(p, j)=$ $\left[\log _{p} r_{p}(j)\right]$, where $[x]$ here denotes the greatest integer less than $x$, and $\log _{p}$ means logarithm to base $p$; then $\alpha(j)=\Pi_{p} p^{a(p, j)} \cdot \beta(j)$ will be the least common multiple of the numbers no greater than $j . \quad \gamma(j)$ is the product of primes no greater than $j$, and $\delta(j)=\gamma(j) \gamma([j / 2])^{-1}$. We note that $\delta(j)$ divides $\gamma(j)$ divides $\beta(j)$ divides $j$ !, and $\alpha(j)$ divides $\beta(j)$ divides $j$ ! Also note $\beta(j)$ divides $\beta(j+1)$, and similarly for $\gamma$, but not for $\delta$ or $\alpha$.

C. H. may now be written in the form $z=\log \left(\prod_{i=1}^{n} \exp y_{i}\right)=$ $\sum_{j=1}^{k}(j ! \alpha(j-1))^{-1} S_{j}$, where $S_{j}$ is an integral sum of commutators in the $y_{i}^{\prime}$ 's, with $S_{1}=\sum_{i=1}^{n} y_{i}$. C. H. is the main tool in linearizing the properties of nilpotent groups, that is, relating structure in $\mathscr{N}_{k}(X, A)$ to structure in $\mathscr{L}_{k}(X, A)$. Let $G$ be a subgroup of $\mathscr{N}_{k}(X, A)$, and $\widetilde{L}=\log G$ its inverse image in $\mathscr{L}_{k}(X, A)$. Let $L$ be the additive subgroup generated by $\tilde{L}$. By the method outlined in [5], for the proof of Proposition 0 , it may be shown that $k ! \alpha(k-1) L \subseteq \widetilde{L}$, and in fact, as $k$ increases this estimate gets worse. A much simpler calculation shows that if $y \in \mathscr{L}_{k}$, and $\exp y$ normalizes $G$, then $[y, L] \subseteq$ $\beta(k-1)^{-1} L$. These facts allow one to use C. H. effectively in establishing properties of nilpotent groups. Here are some typical applications, which will be useful later.

Lemma 1. Let $N$ be a k-step nilpotent group, generated by a subset $X \subseteq N$, consisting of torsion elements, whose orders are all bounded by $n$. Then $N$ consists of torsion elements, of uniformly bounded order, depending only on $k$ and $n$. Also if $\sharp(X)$ (the cardinality of $X$ ) is finite, so is $\#(N)$.

Proof. In $\mathscr{N}_{k}(X, Q)$, consider $G$, the group generated by $\left\{\exp X_{i}: x \in X\right\}$. As we have said, $G \cong N_{k}(X)$. Let $\widetilde{L}=\log G$, and let $L$ be the additive subgroup of $\mathscr{L}_{k}$ generated by $\widetilde{L}$. Let $G^{\prime}$ be the group generated by $\left\{\exp n ! X_{i}\right\}$, and let $\widetilde{L}^{\prime}, L^{\prime}$ be analogous to $\tilde{L}, L$.

It follows easily from the two facts stated above that any commutator in the $X_{i}$ is contained in $n !^{-k} \beta(k-1)^{-k} L^{\prime}$. From this, it follows that for $z \in G, n !^{k} \beta(k-1)^{k} k !^{2} \alpha(k-1)^{2}$ is a bound on the order of the elements of $G / G^{\prime}$ and hence of $H$, since $H$ is a homomorphic image of $G / G^{\prime}$. Since, if $X$ is finite, $\mathscr{L}_{k}(X, Q)$ is finite dimensional over $Q, G / G^{\prime}$ is finite in this case. 
COROLLARY. In any nilpotent group, the torsion elements form a characteristic subgroup.

LEMMA 2. In a nilpotent group, any torsion element commutes with any divisible element.

Proof. Consider, in $N_{k}(x, y) \subseteq \mathscr{N}_{k}(x, y, Q)$, the normal subgroup $G$ generated by $\exp y$, and $G^{\prime}$, the normal subgroup generated by $\exp n y$ for some $n$, and let $\widetilde{L}, L, \tilde{L}^{\prime}, L^{\prime}$ be as usual. Since $\exp x$ normalizes $G$, it follows that if $z=n^{k} \beta(k-1)^{k+1} k !^{2} \alpha(k-1)^{2} x$, then $[z, L] \subseteq k ! \alpha(k-1) L^{\prime} \subseteq \widetilde{L}^{\prime}$. Hence in $N_{k}(x, y) / G^{\prime}$, exp $z$ centralizes $y$. Since the general situation is again a homomorphic image of this one, the lemma follows.

Lemma 3. In a nilpotent group, the divisible elements form a characteristic subgroup.

Proof. If they form a subgroup, it is certainly characteristic. Put now $G=N_{k}(x, y) \subseteq \mathscr{N}_{k}(x, y, Q)$ with $G^{\prime}$ generated by $\exp n x$, $\exp n y$. The $L$ 's as before. By reversing the estimate of Lemma one, we see that $\tilde{L}^{\prime} \subseteq n^{k} \beta(k-1)^{-k} k !^{-2} \alpha(k-1)^{-2} \widetilde{L}$, and from this it is easy to see that the product of divisible elements is divisible. In fact, the set of elements divisible by any given set of primes forms a characteristic subgroup.

We now wish to investigate the existence of lie algebras for groups $G \subseteq \mathscr{N}_{k}(X, A)$. Let $L \subseteq \mathscr{L}_{k}(X, A)$ be any additive subgroup, and let $L^{(j)}$ be the additive subgroup generated by all $j$ th order brackets of elements in $L$. The form of C. H. shows that in order that $\exp L$ be a subgroup of $\mathscr{N}_{k}(X, A)$, it suffices that $L^{(j)} \subseteq$ $j ! \alpha(j-1) L$. A single condition which guarantees this is $L^{(2)} \cong$ $2 \gamma(k) L$. This condition is convenient to work with, so we focus attention on it. An $L$ with this property, and also the group $\exp L$, will be called e.e., short for elementarily exponentiable. If $G$ is a general subgroup of $\mathscr{N}_{k}(X, A)$, with $\widetilde{L}, L$ as usual, then $L^{(2)} \subseteq$ $\beta(k-1)^{-1} L$. It follows by simple calculation, using $k ! \alpha(k-1) L \subseteq \widetilde{L}$, that there are $L_{1}$ and $L_{2}$, both e.e., such that $(2 \gamma(k) \beta(k-1))^{k} L_{2} \subseteq L$, and $L_{1}=k ! \alpha(k-1) \delta(k) L$. Since $L_{1} \leqq L$, we note that the general subgroup $G$ of $\mathscr{N}_{k}(X, A)$ contains a normal e.e. subgroup such that the quotient is a torsion group, with elements of uniformly bounded order, the bound depending only on $k$.

Now, if $L \subseteq \mathscr{L}_{k}$ is e.e., then $\exp L=G$ can be considered to have a lie algebra, namely $L$. Then exp: $L \rightarrow G$ is a bijection, with log as inverse, satisfying the Campbell-Hausdorff formula, in the sense that $\log (\exp x \exp y)$ is given as the appropriate sum of com- 
mutators in $x$ and $y$, as indicated by C. H. This is in fact a situation with intrinsic meaning, verifiable in an abstract setting, as we now show. Let $L$ be any abelian group equipped with a bracket operation which is anti-symmetric and satisfies the Jacobi identity, and which, therefore, may be considered a lie algebra. Suppose $L$ is $k$-step nilpotent, i.e., every $(k+1)$ st order commutator vanishes. If $[L, L] \supseteqq$ $2 \gamma(k) L$, and if $L$ has no $p$-torsion for primes $p$ less than $k+1$, then we will say $L$ is e.e.

Proposition 1. If $L$ is an e.e. k-step nilpotent lie algebra, then there is a k-step nilpotent group $G$, and a bijection exp: $L \rightarrow G$, with inverse log, satisfying the Campbell-Hausdorff formula. G and exp are unique up to isomorphism.

Proof. Since C. H. determines the group law, uniqueness is clear. Let $A_{0}$ be the smallest subring of $Q$ which contains $Z$, and in which division by $k$ ! is permissible. Consider $L$ as a $Z$-module, and form $L^{\prime}=L \otimes_{z} A_{0}$. By our assumption on the torsion properties of $L$, the natural map $L \rightarrow L^{\prime}$ is monomorphic, so we may consider $L$ as a subalgebra of $L^{\prime}$. For an appropriate $X$, there is a surjection $\sigma: \mathscr{L}_{k}\left(X, A_{0}\right) \rightarrow L^{\prime}$. Consider $\sigma^{-1}(L)$. If $a, b \in \sigma^{-1}(L)$, then since $L$ is e.e., there is $c$ in $\sigma^{-1}(L)$ such that $[a, b]-2 \gamma(k) c=d \in \operatorname{ker} \sigma$. Since $\operatorname{ker} \sigma$ is an $A_{0}$-module, $d^{\prime}=2 \gamma(k)^{-1} d \in \operatorname{ker} \sigma$, so $[a, b]=2 \gamma(k)\left(c+d^{\prime}\right)$, so $\sigma^{-1}(L)$ is an e.e. subalgebra of $\mathscr{L}_{k}\left(X, A_{r}\right)$, and $\operatorname{ker} \sigma \subseteq \sigma^{-1}(L)$ is an ideal, and, since ker $\sigma$ is an $A_{0}$-module, it is trivial that $\left[\sigma^{-1}(L), \operatorname{ker} \sigma\right] \subseteq$ $2 \gamma(k) \operatorname{ker} \sigma$. Put now $G_{1}=\exp \sigma^{-1}(L) . \quad G_{2}=\exp \operatorname{ker} \sigma$. Then $G_{1}, G_{2}$ are subgroups of $\mathscr{N}_{k}\left(X, A_{0}\right), G_{2}$ is normal in $G_{1}$, and moreover the relation just above shows $\log \left(g G_{2}\right)=\log g+\log G_{2}$, for any $g \in G_{1}$. It follows that exp factors to a map from $L=\sigma^{-1}(L) / \operatorname{ker} \sigma$ to $G_{1} / G_{2}$, and this obviously satisfies C. H. This establishes existence.

The above proof suggests a more general situation. If $L$ is an e.e. subalgebra of $\mathscr{L}_{k}(X, A)$, and $L^{\prime} \subseteq L$ is a sublattice, such that $\left[L, L^{\prime}\right] \subseteq 2 \gamma(k) L^{\prime}$, we will say $L^{\prime}$ is e.e.-embedded in $L . L^{\prime}$ is then $a$ fortiori e.e., and $G^{\prime}=\exp L^{\prime}$ is a normal subgroup of $G_{1}$ and also $\log \left(g G^{\prime}\right)=\log g+\log G^{\prime}$, so that exp factors to a well defined bijection exp: $L / L^{\prime} \rightarrow G / G^{\prime}$. If $L / L^{\prime}$ has no $p$-torsion for primes $p<k+1$, then we are in fact in the situation of Proposition one, and may say exp satisfies C. H. and is essentially unique. However, if there is $p$ torsion for $p \leqq k$, it no longer makes sense to say exp satisfies C. H. It has certain nice properties: for example, it is a homomorphism from abelian subalgebras of $L / L^{\prime}$ to abelian subgroups of $G / G^{\prime}$; and any e.e. subalgebra of $L$ factors to a subalgebra whose image under exp is a subgroup of $G / G^{\prime}$; but its uniqueness, the extent to which 
it is canonical, is unclear. Nevertheless, it is desirable to have even a noncanonical lie algebra for a group. In this connection we have

Proposition 2. Let $N$ be a k-step nilpotent group. Then there is a torsion-free k-step nilpotent group $G$, and a surjective homomorphism $h: G \rightarrow N$, with the property that there are two normal, e.e. subgroups $M_{1}, M_{2}$ of $G$, with $G \supseteqq M_{1} \supseteqq M_{2} \supseteqq(\operatorname{ker} h) \cap M_{1}$, and with $M_{2}$ e.e.-embedded in $M_{1}$, and such that $G / M_{1}$, and $M_{2} / \operatorname{ker} h \cap M_{1}$ are torsion groups, all elements of which have order dividing $k ! \alpha(k-1) \delta(k)$.

REMARK. Thus long thin relatively commutative groups can be linearized fairly successfully, while short, fat, relatively noncommutative groups cannot.

Proof. For a suitably large set $X$, consider $\mathscr{N}_{k}(X, Q)$, and choose a subgroup $G$ isomorphic to $N_{k}(X)$, and let $h: G \rightarrow N$ be any surjective homomorphism. Let $\tilde{L}, L$ be related to $G$ as usual, and put $L_{1}=$ $k ! \alpha(k-1) \delta(k) L$. Let $L^{\prime}$ be the lattice generated by $\log \operatorname{ker} h$, and put $L_{2}=L^{\prime} \cap L_{1}$. Let $M_{1}=\exp L_{1}$, and $M_{2}=\exp L_{2}$. As we remarked above, $L_{1}$ is e.e.; it is clearly invariant under conjugation by $G$. Also $\left[L, L^{\prime}\right] \subseteq \beta(k-1)^{-1} L^{\prime}$. Hence $\left[L_{1}, L_{2}\right] \subseteq 2 \gamma(k) L_{2}$, for the same reason that $L_{1}$ is e.e. So $M_{2}$ is a group, normal and e.e.-embedded in $M_{1}$. The rest is clear.

We thus have the following corollary essentially established in $[5]$.

CoRollary. A p-group, for any prime $p>k$, has a uniquely defined lie algebra.

Thus, by using e.e. lie algebras, we may satisfactorily linearize a large number of nilpotent groups. This procedure, however, has the disadvantage that it begins with lie algebras and constructs the corresponding groups. It would be desirable to have an intrinsic description of groups which are satisfactorily linearizable in the above manner. The last corollary provides one answer to this problem. Another, still crude, answer is contained in the following results.

Proposition 3. Let $G$ be a k-step nilpotent group, with no $p$ torsion for primes $p \leqq k$. Then in order for $G$ to be e.e., it is sufficient that every commutator $(x, y)$ in $G$ be $z^{m}$ for some $z \in G$, where $m=2 \gamma(k)($ l.c.m. $\{j ! \alpha(j-1): j \leqq k\})$.

Proof. First suppose $G \subseteq \mathscr{N}_{k}(X, A)$ for some $X$ and $A$, and let 
$\tilde{L}, L$ be as usual. Using C. H., particularly the case of it noted in the appendix of [5] regarding commutators, one may show that the above assumption on commutators implies $[L, L] \subseteq L$. Then, the same process, by bootstrapping, implies $[L, L] \subseteq 2 \gamma(k) L$, so $L$ is in fact e.e. It follows that $L_{1}=k ! \alpha(k-1) L$ is e.e.-embedded in $L$. Since $G^{(2)} \subseteq L_{1}$ by assumption, a second bootstrap shows that in fact $[L, L] \subseteq L_{1}$. We also have, of course, that $L_{1} \subseteq \widetilde{L}$. We now have that $\log (x y)=\log x+\log y$ (modulo $L_{1}$ ), and from this, it is immediate that $\log x+\log y \in \widetilde{L}$, so $\widetilde{L}=L$.

For general $G$, take $\mathscr{N}_{k}\left(X, A_{0}\right)$ as in Proposition one, choose $G_{0} \subseteq$ $\mathscr{N}_{k}\left(X, A_{0}\right)$, isomorphic to $N_{k}(X)$, and let $h: G_{0} \rightarrow G$ be a surjective homomorphism. Let $M$ be the $A_{0}$ submodule of $\mathscr{L}_{k}(X, A)$ spanned by $\log \operatorname{ker} h$. Then it is easy to see that $\exp M$ is a subgroup of $\mathscr{N}_{k}\left(X, A_{0}\right)$, normalized by $G_{0}$. Since $y \in M \Rightarrow k !^{n} y \in \log$ ker $h$, we see that our restriction on the torsion properties of $G \operatorname{imply} \exp M \cap G_{0}=$ ker $h$. Therefore, if $G_{1}$ is the subgroup generated by $\exp M$ and $G_{0}$, there is a unique extension $h_{1}$ of $h$ to $G_{1}$, such that $\operatorname{ker} h_{1}=\exp M$. Now, our assumption on $G$ implies, for $x, y \in G_{1},(x, y)$ is the $2 k ! \alpha(k-$ 1) $\gamma(k)$ th power of $z$, modulo $\exp M$. But since $M$ is an $A_{0}$-module, $G_{1}$ actually satisfies the condition also. Finally, we see $\exp M$ is e.e.embedded in $G_{1}$, so $h_{1}: G_{1} \rightarrow G$ defines an e.e. linearization of $G$.

Proposition 4. If $G \subseteq \mathscr{N}_{k}(X, A)$ is any subgroup, with $\tilde{L}, L$ as usual, and if $L_{1}=k ! \alpha(k-1) \gamma(k) L$, then $G_{1}=\exp L_{1}$ is exactly the subgroup $G^{\prime}$ of $G$ generated by the $k ! \alpha(k-1) \gamma(k)$ th powers of elements of $G_{1}$.

Proof. Obviously $G_{1} \supseteq G^{\prime}$. On the other hand $\log G^{\prime}$ clearly generates $L_{1}$ as additive group. Hence $\left[L_{1}, L_{1}\right] \subseteq k ! \alpha(k-1) L_{1} \subseteq$ $\log G^{\prime} . \quad k ! \alpha(k-1) L_{1}$ being amply e.e.-embedded in $L_{1}$, the same procedure as the final step of Proposition 3, in the case $G \cong \mathscr{N}_{k}(X, A)$, shows $\log G^{\prime}$ is actually closed under addition, and so equal to $L_{1}$.

II. Topological structure of nilpotent groups. A good place to start, when analyzing a class of topological groups, is with the connected ones. Recall ([4], Theorem 9.8) that a connected locally compact abelian group is of the form $R^{n} \times C$, where $C$ is connected and compact.

LEMMA 4. If $N$ is a connected, nilpotent locally compact group, and $\mathscr{Z}(N) \cong R^{n} \times C$, then $\tilde{N}=N / C$ is a simply connected lie group.

Proof. Put $Q_{0}=\mathscr{Z}(N) / C, Q_{i}=\mathscr{Z}^{i+1}(N) / \mathscr{Z}^{i}(N)$ for $i \geqq 1$. It suffices to show that $Q_{i}$ is isomorphic to $R^{n_{i}}$. This is true by 
definition for $i=0$. The situations being entirely parallel for $i \geqq 1$, it suffices to consider $Q_{1}$. We must show that the compact part of $Q_{1}$ is trivial. Let $x \in Q_{1}$ generate a group $A$ with compact closure. If $x$ is not the identity then $(x, N) \subseteq \mathscr{z}(N)$ is a nontrivial subgroup. Choose any $\chi \in \hat{\tilde{y}}$, such that $(x, N) \nsubseteq$ ker $\chi$. Then, for each $n \in N, \chi_{n}(y)=\chi((y, n))$ for $y \in A$ defines a character on $A$; and the map $n \rightarrow \chi_{n}$ is a homomorphism from $N$ to $\hat{A}$, which is nontrivial by construction. But this is impossible, because $N$ is connected and $\hat{A}$ is discrete.

Then it is nice to know how the connected component of the identity sits in a nonconnected group.

Lemma 5. Let $N$ be a locally compact nilpotent group, $N_{0}$ the identity component of $N$, and $D$ the centralizer of $N_{0}$. Then $N / D=M$ is torsion-free, and $N_{0} / \mathscr{F}_{\bar{z}}\left(N_{0}\right)=M_{0}$ is open in $M$.

Proof. Take any $m \in M$. Then $m$ defines an automorphism $m$ of $N_{0}$. Let $C$ be the compact part of $\mathscr{z}\left(N_{0}\right)$. Then Ad $m$ leaves $C$ stable. (In fact, $C$ is "topologically characteristic" in $N_{0}$.) Let $C^{1}(m)$ be the subgroup of $C$ centralized by $m$, and $C^{2}(m)$ the subgroup of elements left fixed modulo $C^{1}(m)$. $N$ being nilpotent, $C^{1}(m)$ is nontrivial, and if $C^{1}(m) \neq C$, so is $C^{2}(m) / C^{1}(m)$. By inspection of the action of $\mathrm{Ad}^{*} m$ on $C^{2}(m)$ (which is torsion-free since $C$ is connected) we see it is torsion-free if it is nontrivial. If it is trivial, then $C^{1}(m)=C$, and $\mathrm{Ad}^{*} m$ defines a unipotent automorphism of the lie algebra of $N_{0} / C$, which again cannot be a torsion element.

To show $M_{0}$ is open in $M$, it suffices to show $M / M_{0}$ is discrete. But since $M / M_{0}$ is totally disconnected, if it is not discrete, it contains a nontrivial compact subgroup ([4], p. 61). Let $m \notin M_{0}$ be in the inverse image of $M$ of such a subgroup. Then $C^{1}(m)=C$. For otherwise, let $A$ be the closed group in $M / M_{0}$ generated by $m$. Then for $a \in A$, and any $\chi \in \widehat{C^{1}(m)}, a \rightarrow \chi(a)$, given by $\chi(a)(c)=\chi((a, c))$, for $c \in C^{2}(m)$, defines a homomorphism $A \rightarrow \widehat{C^{2}(m)}$, which must be trivial, since $A$ is compact, but $\widehat{C^{2}(m)}$ is discrete and torsion-free. Therefore $C^{2}(m)=C^{1}(m)=C$. Hence Ad $m$ factors to a unipotent automorphism of the lie algebra of $N_{0} / C$, which normalizes $\operatorname{Ad} M_{0}$, and so generates with Ad $M_{0}$ a closed group of automorphisms of $N$, of which Ad $M_{0}$, the identity component, is open. This is again inconsistent with the hypothesis on $m$, so $M / M_{0}$ must actually be discrete.

Corollary. If $n \in N$, and the image of $n$ in $N / N_{0}$ is a quasitorsion element, then there is $n_{0} \in N_{0}$, such that $\operatorname{Ad} n$ and $\operatorname{Ad} n_{0}$ agree on $N_{0}$. 
Proof. The hypothesis on $n$ implies, by Lemma 5, that the image of $n$ in $M / M_{0}$ is the identity.

The next step is to analyze totally disconnected groups.

LEMma 6. Let $N$ be a totally disconnected, nilpotent, locally compact group. Let $H_{1}, H_{2}$ be any two compact subgroups. Then $H_{3}$, the subgroup generated by $H_{1}$ and $H_{2}$, is also compact.

Proof. Since $N$ is totally disconnected, and $H_{1}$ and $H_{2}$ are compact, by induction on the nilpotent length (see Lemma 8), there is a compact open subgroup $M$ normalized by $H_{1}$ and $H_{2}$. We may clearly assume $M \subseteq H_{i}, i=1,2$. Then $M$ will be a compact open normal subgroup of $H_{3}$ and $H_{3} / M$ will be generated by $H_{1} / M$ and $H_{2} / M$, which are finite torsion groups. By Lemma one, $H_{3} / M$ is also finite, so $H_{3}$ is compact.

COROLLARY. The set of quasitorsion elements form an open normal subgroup of $N$.

Proof. That they form an open set follows from the existence of open compact subgroups. That they are a normal set is obvious. That they are a subgroup follows from the lemma.

Denote by $N_{c}$ the subgroup of quasitorsion elements of totally disconnected nilpotent $N$.

COROLLARY. $\quad N_{c}$ is expressible as an increasing union (inductive limit) of open compact subgroups.

Of course, if $N_{c}$ is separable, it is the union of a countable increasing sequence of compact open subgroups.

If $M \subseteq N_{c}$ is open and compact, then $M$ is pro-finite, and as such has Sylow $p$-subgroups $M_{p}([11])$. The basic structural fact for finite nilpotent groups ([3]) carries over in the projective limit to imply that each $M_{p}$ is unique and normal, and $M$ is the direct product of the $M_{p}$ 's. Define $N_{c}(p)$ to be the union of the $M_{p}$ 's as $M$ ranges over all open compact subgroups; then $N_{c}(p)$ will be a closed normal subgroup. The following is now clear.

LEMma 7. If $M \subseteq N_{c}$ is any open compact subgroup, then $N_{c}$ is the restricted direct product of the $N_{c}(p)$ 's with respect to the $M_{p}$ 's.

Now we show that, although open compact subgroups of $N_{c}$ are not normal, they almost are.

Lemma 8. Let $M \cong N_{c}$ be open and compact, let $n \in N$. Then 
some power of $n$ normalizes $M$. Reciprocally, given a finite number of elements $n_{1}, \cdots, n_{l}$ of $N$, there are open compact subgroups $M_{1} \subseteq$ $M \subseteq M_{2}$, with $M_{i}$ normalized by $n_{j}$.

Proof. We prove the second statement first. Certainly, there are subgroups of $\mathscr{z}(N)$, contained in and containing $\mathscr{z}(M)$, which are normalized by $n_{1}, \cdots, n_{l}$. Assume that, given any compact neighborhood $U$ of the identity, there are compact, relatively open subgroups $M_{1}^{i}, M_{2}^{2}$ of $\mathscr{F}^{i}(N)$, contained in and containing $U$, and normalized by $n_{1}, \cdots, n_{l}$. Then suppose $M_{1}^{i+1}, M_{2}^{i+1}$ are relatively open groups of $\mathscr{Z}^{i+1}(N)$, such that $M_{j}^{i+1} \cap \mathscr{\not}^{i}=M_{j}^{i}$. In order for $M_{j}^{i+1}$ to be normalized by $n_{1}, \cdots, n_{k}$, it is necessary and sufficient that $\left(n_{h}, M_{j}^{i+1}\right) \subseteq$ $M_{j}^{i}$ for $1 \leqq h \leqq l$. But since taking commutators is continuous, we clearly can choose $M_{1}^{i+1}$ small enough to accomplish this; and on the other hand, if we choose $M_{2}^{i}$ big enough, we may also make $M_{2}^{i+1}$ very big. By induction then, we may find $M_{1}, M_{2}$ as desired.

For the first part, given $M$ and $n$, we choose $M_{1} \subseteq M \cong M_{2}, M_{i}$ normalized by $n$. Then Ad $n$ factors to an automorphism of $M_{2} / M_{1}$, some power of which must be trivial; this power will in particular normalize $M$.

Corollary 1. A totally disconnected, separable, locally compact nilpotent group $N$ may be expressed as the union (inductive limit) of an increasing sequence of open subgroups $N_{i}$, such that each $N_{i}$ contains a compact subgroup $K_{i}$, such that $K_{i}$ is open in $N$, and $N_{i} / K_{i}$ is discrete and torsion-free, and arbitrarily small neighborhoods of the identity contain normal open subgroups.

Proof. Since $N$ is separable, $N / N_{c}$ is countable. Choose a sequence $\left\{n_{i}\right\}_{i=1}^{\infty}$ which generate $N$-modulo $N_{c}$. Let $M_{j}$ be a compact open subgroup of $N$, normalized by $\left\{n_{i}\right\}_{i=1}^{j}$. Then in the group generated by the $\left\{n_{i}\right\}_{i=1}^{j}$, the subgroup contained in $N_{c}$ is, modulo $M_{j}$, a finitely generated ([3], p. 153) torsion group.

Then choosing $K_{j}$ large enough to contain $M_{j}$ and this subgroup too, and to be normalized by $\left\{n_{i}\right\}_{i=1}^{j}$, we get, provided we pick the $K_{j}$ 's big enough that they exhaust $N_{c}$, the desired sequence.

COROLlaRY 2. All locally compact nilpotent groups are unimodular.

Proof. This is immediate from Lemma 8 for totally disconnected groups. But on the connected component $\operatorname{Ad} n$, for $n \in N$, acts as a unipotent, hence measure-preserving automorphism. The combination of these facts quickly gives the corollary. 
We now give closer attention to the Sylow, quasi-p groups. The next lemma is the analogue for quasi- $p$ groups of Lemma 4.

Lemma 9. Let $N_{p}$ be a nilpotent quasi-p group. An element $x \in N_{p}$ is divisible if and only if it is divisible by $p$. Let $D$ be the subgroup of divisible elements. Then if $D$ is torsion-free, division by $p$ is continuous on $D$ if and only if $D$ is closed. In this case, $D$ is isomorphic to a unipotent algebraic group over $Q_{p}$ (the $p$-adic numbers).

REMARK. By a unipotent algebraic group over $Q_{p}$, we will always understand the $Q_{p}$-rational points of such a group with the locally compact topology.

Proof. Any $x \in N_{p}$ is contained in a compact subgroup $K$. If $K^{\prime}$ is an arbitrary small open normal subgroup of $K$, then $K / K^{\prime}$ is a $p$-group, so if $n$ is prime to $p, x \rightarrow x^{n}$ induces a bijection on $K / K^{\prime}$. Hence $x$ has an $n$th root modulo $K^{\prime}$. In the limit, it has an $n$th root. Suppose now $D$ is torsion-free, and division by $p$ is continuous. Without loss of generality, we may assume $D$ is dense. In this case, consider $\mathscr{Z}(D)=\mathscr{Z}(N) \cap D$. This is then an abelian group, and Lemma 2 shows it is preserved by division by $p$. Hence restriction of division by $p$ to $\mathscr{Z}(D)$ is continuous. Thus, given a compact neighborhood $V$ of the identity, there is a compact open subgroup $U$ such that all $p$ th roots of elements of $U \cap \mathscr{Z}(D)$ are in $V$. Now if $x \in \overline{\mathscr{Z}(D)}$ (closure), then we may find $y \in \mathscr{Z}(D)$, such that $y-x \in U$. Let $\left\{Z_{\alpha}\right\}$ be a net in $\mathscr{z}(D) \cap U$ approaching $y-x$. Then $\left\{Z_{\alpha} / p\right\}$ has a cluster point $q$ in $V$, which, by continuity of taking $p$ th powers, must be $p$ th root of $y-x$. Thus $p((y / p)-q)=x$, so $x$ is divisible by $p$. Similarly $x$ is divisible by $p^{n}$ for any $n$. Hence $\mathscr{Z}(D)$ is a closed subgroup of $\mathscr{Z}(N)$. Now consider the projection $\pi: N \rightarrow N / \mathscr{Z}(D)=N^{\prime}$. If $D^{\prime}$ is the group of divisible elements of $N^{\prime}$, then it is easy to verify $D^{\prime}=\pi(D)$. But now $N^{\prime}$ is of smaller nilpotent length than $N$; so by induction, we may assume $D^{\prime}=N^{\prime}$. Then one quickly sees that $D=N$ also.

On the other hand, suppose $D$ is closed. It is then a divisible torsion-free nilpotent quasi- $p$ group. Now on any abelian subgroup of $D_{1}$ taking some power is a homomorphism. We see that in particular $\mathscr{Z}(D)$ is a $Z$-module; and the topology is such that it is in fact a (locally compact) $Q_{p}$-module. It is well known ([12]) then that $\mathscr{Z}(D)$ is isomorphic to $Q_{p}^{m}$ for some $m$. Dividing out by $\mathscr{Z}(D)$, we may assume by induction that $D / \mathscr{Z}(D)$ is a unipotent algebraic group over $Q_{p}$. $D$ being a central extension of $D / \mathscr{z}(D)$ by $Q_{p}^{m}$, it clearly is such also. Division by $p$ is then clearly continuous on $D$. 
CoROllaRY. If $N$ is a divisible locally compact nilpotent quasi-p group, and $T$ is the subgroup of torsion elements, then $T \subseteq \mathscr{F}(N)$, and $N / T$ is an algebraic unipotent group over $Q_{p}$.

Recall a topological group is finitely generated if it has a finitely generated dense subgroup.

LEMMA 10. Let $M$ be a compact, torsion-free nilpotent quasi-p group. Then $M$ is isomorphic to an open subgroup of a unipotent algebraic group over $Q_{p}$ if and only if $(M)^{p}$ is open in $M$ if and only if $M$ is finitely generated.

Proof. The first condition clearly implies the second and third conditions. On the other hand, from the work of Malcev ([1]), or from $\S 1$, a finitely generated, torsion-free, nilpotent discrete group $\Gamma$ is canonically embeddable in a unipotent algebraic group $N_{Q}$ over $Q$. Giving $N_{Q}$ the $p$-adic topology, and completing, $\Gamma_{p}$, the completion of $\Gamma$ becomes a compact group, and the natural inclusion $\Gamma \rightarrow M$ extends to a surjective homomorphism $h: \Gamma_{p} \rightarrow M$. Since $M$ is torsion-free, we see that $H$, the smallest divisible group of $N_{Q_{p}}$ (the $p$-adic completion of $N_{Q}$ ) containing $\operatorname{ker} h$, is normal in $N_{Q_{p}}$, and also $H \cap \Gamma=\operatorname{ker} h$, so that $M$ is naturally isomorphic to $\Gamma_{p} / \operatorname{ker} h \cong N_{Q_{p}} / H$. Finally, we see that, if $M$ is abelian, then taking $p$ th powers is a homomorphism, and if $p M$ is open, then actually $M$ is a finitely generated $\boldsymbol{Z}_{p}$ ( $p$-adic integers)-module, and so is finitely generated. But clearly the category of finitely generated pro-p groups permits group extensions. Hence, by induction on nilpotent length, nilpotent pro-p groups $M$ such that $M^{p}$ is open are finitely generated.

REMARK. Of course, unipotent algebraic groups over $Q_{p}$ are the most interesting examples of nilpotent quasi- $p$ groups. Their representation theory is in fact already known ([8]). The above lemmas indicate the role they play in the class of all quasi- $p$ nilpotent groups.

At this point, we note that by a straightforward process of taking a projective, then an inductive limit, Theorem I (stated in the introduction) follows from Proposition one and the corollary to Proposition two.

III. Harmonic analysis. We begin with a result which is in fact independent of lie algebras, and holds for a general separable locally compact nilpotent group. First we make some definitions. If $G$ is separable and locally compact, $H \subseteq G$ a closed subgroup, then we have certain maps $r$ and $i$, from ideals in $C^{*}(G)$ to ideals in $C^{*}(H)$, and vice versa. If $I \subseteq C^{*}(G)$ is a closed two-sided *-ideal, 
let $I^{\perp} \leqq C(G)$ be the cone of all continuous positive definite functions which, considered as elements of the dual of $C^{*}(G)$, annihilate $I$. We call $I^{\perp}$ the dual ideal associated to $I$. Let $r(I)$ be the ideal in $C^{*}(H)$ annihilated by the restrictions to $H$ of all functions in $I^{\perp}$. Then $r(I)^{\perp}$ is the set of all $f \in C(H)$ which are uniform-on-compacta limits of restrictions of $g \in I^{\perp}$. Reciprocally, starting with $J$, a closed, two-sided *-ideal of $C^{*}(H)$, take $J^{\perp} \subseteq C(H)$, and identify $f \in J^{\perp}$ with the measure $f d h$ on $G$, where $d h$ is Haar measure on $H$. Then consider all positive definite functions in $C(G)$ which are uniform-oncompacta limits of (positive) sums of functions in $C(G)$ of the form $a * f d h * a^{*}$, where $a \in C_{0}(G), a * b$ indicates convolution, and $a^{*}$ is the adjoint of $a$ (as an element of $C^{*}(G)$ ). These functions make a cone, which is $I^{\perp}$ for some two-sided closed *-ideal $I$ of $C^{*}(G)$, and we define $I=i(J)$.

These maps are simply the extensions to general groups of the $r$ and $i$ of [5]. They have the same properties. Specifically, if $U$ is a representation of $G$, with kernel $I$ in $C^{*}(G)$, and if the restriction of $U$ to $H$ has kernel $J$ in $C^{*}(H)$, then $J=r(I)$. Reciprocally, if $V$ is a representation of $H$, with kernel $J$ in $C^{*}(H)$, and if the representation of $G$ induced by $V$ has kernel $I$ in $C^{*}(G)$, then $I=i(J)$. Also, $i(r(I)) \subseteq I$ if $G$ is amenable, and $r(i(J)) \subseteq J$. If $i(r(I))=I$, we say $I$ is induced from, or may be reduced to, $H$.

Of particular importance is the case $H$ when is normal, and $I$ is primitive. Then conjugation in $G$ induces an action $\mathrm{Ad}^{*} G / H$ of $G$ ( $H$ acts trivially) on $M(H)$, the primitive ideal space of $H$. If $I \in M(G)$, i.e., if $I$ is primitive, then $r(I)$ is an $\mathrm{Ad}^{*} G / H$ quasiorbit in $M(H)$, so $r$ defines a map $r: M(G) \rightarrow M(H ; G)$, the quasiorbit space of $M(H)$ by $\mathrm{Ad}^{*} G / H$, which we call the relative primitive ideal space of $H$ in $G$, by virtue of this map r.r is surjective and continuous.

Proposition 5. Let $N$ be a separable, locally compact $k$-step nilpotent group, and $I \in M(N)$. If $I$ corresponds to a faithful represention of $N$, of degree not one, then $I$ can be reduced to $a$ $(k-1)$-step nilpotent normal subgroup. In any case, there is a closed subgroup $M$, and a one-dimensional character $\chi$ of $M$ such that the representation $U^{x}$ of $N$ is irreducible, with kernel $I$ in $C^{*}(N)$.

Proof. Let $U$ be any representation of $N$ with kernel $I$ in $C^{*}(N)$. Then $U$ defines a character $\psi$ on $\mathscr{Z}(N) . \psi$ depends only on $I$, and by our assumption on $\mathrm{I}$, it is faithful. Consider the pairing $d: \mathscr{z}^{(2)}(N) \times N \rightarrow T$ (the circle) given by $d(z, n)=\psi((z, n)) . \quad d$ in fact factors to a pairing $d: \mathscr{Z}^{(2)}(N) / \mathscr{Z}(N) \times N / N^{(2)} \rightarrow T$. Let $A$ 
be a maximal closed abelian subgroup of $\mathscr{Z}^{(2)}(N)$, and let $N_{1}$ be the centralizer of $A$. Then $N_{1}$ is normal in $N$, and if $N$ is $k$-step nilpotent, $N_{1}$ is $(k-1)$-step nilpotent, because $\mathscr{Z}^{(2)}(N) \cap N_{1}=A \subseteq \mathscr{Z}\left(N_{1}\right)$. Now $d$ becomes a faithful pairing $d: A / \mathscr{Z}(N) \times N / N_{1} \rightarrow T$, and so defines homomorphisms: $d_{1}: A / \mathscr{L}(N) \rightarrow \widehat{N / N_{1}}$ and $d_{2}: N / N_{1} \rightarrow \widehat{A / \mathscr{Y}}(N) . \quad d_{1}$ and $d_{2}$ are injective and have dense images.

Let $\pi: N \rightarrow N / N_{1}$ be the quotient map. If $f \in I^{\perp}$, then a simple calculation shows that, for $a \in A, \operatorname{Ad}^{*} a f(n)=f\left(a n \alpha^{-1}\right)=f(n) d(\alpha, \pi(n))=$ $\overline{f(n) d_{1}(a)(\pi(n))}$ (the bar here denotes complex conjugate). Thus the adjoint action of $A$ on $I^{\perp}$ is simply multiplication by characters of $N / N_{1}$. Since the image of $d_{1}$ is dense, it follows by taking uniformon-compacta limits, that if $\phi$ is any positive-definite function on $N / N_{1}$, then $\varphi f \in I^{\perp}$ whenever $f \in I^{\perp}$. Now let $\left\{\phi_{j}\right\}$ be a positive definite, positive-valued approximate identity in $L_{1}\left(N / N_{1}\right)$. Then if $d n$ is Haar measure for $N, \varphi_{j} d n$ converges, in the weak *topology on measures, to a Haar measure $d n_{1}$ on $N_{1}$. Therefore, if $b \in C_{0}(N), b * \varphi_{j} f * b^{*}$ converges to $b * f_{1} d n_{1} * b^{*}$ uniformly on compacta, where $f_{1}=f_{\mid N_{1}}$. Thus $\left.i\left(r I^{\perp}\right)\right)=I^{\perp}$, and $I^{\perp}$ may be reduced to $N_{1}$, which is the first statement of the proposition.

We prove the second statement by induction on the nilpotent length of $N$. By dividing out by an appropriate subgroup, we may immediately reduce to the case of the first part of the proposition. Use the notation developed there. Then $r(I)$ is an $\mathrm{Ad}^{*} N / N_{1}$ quasiorbit in $M\left(N_{1}\right)$. Let $J \in M\left(N_{1}\right)$ be such that the closure of this orbit is $r(I)$. Then we have $i(J)=I$. By induction, there is a closed subgroup $M$ of $N_{1}$, and a character $\chi$ of $M$, such that the representation $V^{x, M}$ of $N_{1}$ is irreducible, with kernel $J$. Consider the induced representation $U^{x, M}=U^{V N_{1}}$ of $N$. A simple calculation here shows that, under $U^{x}, A$ is mapped to multiplication operators on $N / N_{1}$, exactly as described above. It follows immediately that $U^{x}$ is irreducible. As our previous comments show $U^{x}$ has kernel $I$ in $C^{*}(N)$, this completes the proposition.

From now on, we restrict our attention to a $k$-step nilpotent quasi-p group $\mathscr{P}$, with $p>k$. We notice first that, for $\mathscr{P}$ compact, the main theorem plus explicit multiplicity formulae for tensor products and induced representations follows simply by taking the projective limit of Theorem 3 of [5]. Hence our main task is to cope with the inductive limit necessary to realize a noncompact $\mathscr{P}$. We deal first with the question of attaching ideals to orbits.

Let $P$ be the lie algebra of $\mathscr{P}$. If $R$ is a closed subalgebra of $P$, then we write $\mathscr{R}$ for the subgroup $\exp R$ of $\mathscr{P}$, and vice versa. We use the rest of the notation of the introduction also. Take $\psi \in \hat{P}$. We will say $R$ is a polarizing subalgebra for $\psi$ if $R$ is subordinate to $\psi$ and is a maximal subgroup of $P$ with respect to this property. 
Lemma 11. For any $\psi \in \hat{P}$, there exist polarizing subalgebras $R$ for $\psi$. Moreover, $R$ aan be chosen so that it contains a specified maximal abelian subalgebra of $\mathscr{F}^{(2)}(P)$, and such that $\operatorname{Ad}^{*} \mathscr{R}(\psi)=$ $\operatorname{Ad}^{*} \mathscr{P}(\psi) \cap \psi+R^{\perp}$, this set being dense in $\psi+R^{\perp}$ (here $R^{\perp}$ denotes the annihilator of $R$ in $\hat{P}$ ), and such that $U^{\psi, \mathscr{R}}$ is an irreducible representation of $\mathscr{P}$. (Note $\mathscr{R}$ automatically contains the isotropy group of $\psi$.)

Proof. As usual, we induce on the nilpotent length, and reduce immediately to the case when $\psi$ is faithful on $\mathscr{Z}(P)$. We recall Proposition five, and use the $d, A$, and $\mathscr{P}_{1}\left(=N_{1}\right)$ introduced there. If $P_{1}=\log \mathscr{P}_{1}$, then we can assume by induction that there is $R \subseteq P_{1}$ which polarizes $\psi_{\mid P_{1}}$. We observe that $\log A \cong \mathcal{Z}\left(P_{1}\right)$, so $R \supseteqq \log A$. We may assume $\log A$ is the specified subalgebra of $\mathscr{Z}^{(2)}(P)$. We see if $x \in P$, and $\psi([x, R]) \equiv 1$, then $x \in P_{1}$, since $\log A \subseteq R$, and hence $x \in R$. Similarly if $\mathrm{Ad}^{*} y(\psi) \in \psi+R^{\perp}$ for $y \in \mathscr{P}$, then $y \in \mathscr{P}_{1}$ since $\log A \subseteq R$. But then $\operatorname{Ad}^{*}(y)(\psi)_{\mid P_{1}} \subseteq \psi_{\mid P_{1}}+R_{\mid P_{1}}^{\perp}$, so by induction, and since $\mathscr{R}$ contains the isotropy subgroup of $\psi_{\mid P_{1}} ; y \in \mathscr{R}$. Now $\mathrm{Ad}^{*} A(\psi) \subseteq \psi+P_{1}^{\perp}$, and is dense there; combining this with the parallel fact for $\mathscr{R}$ and $\psi_{\mid P_{1}}$, we see $\operatorname{Ad}^{*} \mathscr{R}(\psi)$ is also dense in $\psi+R^{\perp}$. Finally, if $\psi$ restricted to $\mathscr{R}$ induces an irreducible representation of $\mathscr{P}_{1}$, the calculation of Proposition five shows it also induces an irreducible representation of $\mathscr{P}$.

The above lemma establishes a surjective relation $\alpha$ from $\mathrm{Ad}^{*} \mathscr{P}$ quasiorbits in $\hat{P}$ to $M(\mathscr{P})$. Using the theorem already established for compact groups, we can show the relation $\alpha$ is actually a function, and in fact, bijective and a homeomorphism. In working with the topology of $M(\mathscr{P})$, we use the results of [2], which in our case we may state in the following simple way. Two ideals $I_{1}, I_{2}$ of $C^{*}(\mathscr{P})$ are identical if and only if for every open compact subgroup $M$ of $\mathscr{P}, r\left(I_{1}\right)$ and $r\left(I_{2}\right)$ are the same. Even more directly, $I_{1}, I_{2}$ are the same if and only if, given $U_{i}$, with kernels $I_{i}$, then $U_{i \mid M}$ contain the same irreducible components, for any $M$.

LEMma 12. If $M \cong \mathscr{P}$ is open and compact, let $C \cong P$ be its lie algebra, and let $\pi: \hat{P} \rightarrow \widehat{C}$ be the natural map. Let $V$ be an irreducible representation of $M$ corresponding to an $\mathrm{Ad}^{*} M$ orbit 0 . Take $\psi \in P$, and let $R$ be a polarizing subalgebra for $\psi$, satisfying the conditions of Lemma 11. Let $\theta$ be the $\mathrm{Ad}^{*} \mathscr{P}$ quasiorbit of $\psi$. Then $U_{\mid M}^{\psi, \pi}$ contains $V$ if and only if $\theta \cap \pi^{-1}(0) \neq \varnothing$.

Proof. $M$ being open and compact, the classical criterion [7] for finite groups is valid here also, so $U_{1 M}^{\psi, O}$ contains $V$ if and only if, for some $x \in \mathscr{P}, V$ contains $\mathrm{Ad}^{*} x(\psi)$ restricted to $x \mathscr{R} x^{-1} \cap M$. This 
is the same as to say $\operatorname{Ad}^{*} x\left(\psi+R^{\perp}\right) \cap \pi^{-1}(0) \neq \varnothing$. But $\pi^{-1}(0)$ is clopen, and $\operatorname{Ad}^{*}\left(x \mathscr{R} x^{-1}\right)\left(\operatorname{Ad}^{*} x(\psi)\right)$ is dense in $\mathrm{Ad}^{*} x\left(\psi+R^{\perp}\right)$, by choice of $\mathscr{R}$, so the desired statement follows.

Proposition 6. Let $\theta$ be an $\mathrm{Ad}^{*} \mathscr{P}$ quasiorbit in $\hat{P}$. Take $\psi \in \theta$, let $R$ polarize $\psi$, and satisfy Lemma 11 . Then the correspondence which assigns to $\theta$ the kernel I of $U^{\text {r }}$ in $C^{*}(\mathscr{P})$ defines a natural homeomorphism $\alpha$ between the quasiorbit space $Q(\hat{P})$ and $M(\mathscr{P})$.

Proof. The relation is clearly natural, and we have already seen it is surjective. The remarks preceding Lemma 12 show that lemma characterizes $\alpha(\theta)$ solely in terms of $\theta$, so $\alpha$ is in fact a function. Let $S$ be any set in $Q(\hat{P})$, and $\theta \in Q(\hat{P})$. If $\theta \notin \bar{S}$, there is $\psi \in \theta, \psi \notin \bar{S}$ (regarding $\theta, S$, as subsets of $\hat{P}$ ). Choosing a sufficiently large compact open $M$, we will have $\pi^{-1}(\pi(\psi)) \cap S=\varnothing$. Since $S$ is $\mathrm{Ad}^{*} \mathscr{P}_{\text {-invariant, }}$ this will imply $\pi^{-1}(0) \cap S=\varnothing$, where 0 is the $\mathrm{Ad}^{*} M$ robit of $\pi(\psi)$ in $\log M$ ( $\pi$ of course is the map $\hat{P} \rightarrow \widehat{\log M})$. This establishes immediately that $\alpha$ is one-one and a homeomorphism.

The correspondence being established on the level of point sets, we now examine it in terms of harmonic analysis proper. We have the following generalization of the Bochner theorem on the Fourier transforms of positive definite functions on $L_{1}(G), G$ abelian.

Proposition 7. Via Fourier transform, positive definite Ad* $\mathscr{P}_{-}$ invariant functionals on $\mathscr{A}(\mathscr{P})$ are identified bijectively with $\mathrm{Ad}^{*} \mathscr{P}$-invariant, locally finite, positive measures on $\hat{P}$. The Plancherel measure for $\mathscr{P}$ transforms into Haar measure on $\hat{P}$.

REMARK. A similar theorem has been proven by G. Schiffman [9] for lie groups.

Proof. We know this already for compact groups. The noncompact case is a purely formal matter of taking the inductive limit. We have $\mathscr{A}(\mathscr{P})$ as the inductive limit over $\mathscr{A}(M)$ as $M$ runs through the open compact subgroups of $\mathscr{P}$. The injection $\mathscr{A}(M) \rightarrow \mathscr{A}(\mathscr{P})$ is given by extending $f \in \mathscr{A}(M)$ to be zero outside of $M$. We have also the restriction maps backward. Similarly, if $C \cong \hat{P}$ is open and compact, then $\mathscr{A}(\hat{P})$ is the inductive limit of the subspaces consisting of functions constant on the cosets of $C$. Running through these limits gives the result, since the Fourier transform is natural with respect to the various injections. Since exp preserves Haar measures, and $\mathrm{Ad}^{*} \mathscr{P}$ is a measure-preserving group of automorphisms, the statement about the Plancherel measure follows from the classical Plancherel theorem for abelian groups. 
The above, then, is the broad outline of representation theory for the groups in question. One would, however, like to know some of the details. For example, it seems plausible, after the $p$-adic and compact examples, that there should be exactly one $\mathrm{Ad}^{*} \mathscr{P}_{-}$ invariant measure carried on each $\mathrm{Ad}^{*} \mathscr{P}$ quasiorbit. This is closely related to the question of whether $Q(\hat{P})$ is $T_{1}$, which, by virtue of $\alpha$, is equivalent to all primitive ideals of $C^{*}(\mathscr{P})$ being maximal. The traditional proof, for the real or $p$-adic cases, that the orbits are actually closed does not generalize, and $I$ cannot patch it at present. When $\mathscr{P}$ is actually a discrete torsion group, then $\hat{P}$ is compact, and the distality of $\mathrm{Ad}^{*} \mathscr{P}$, which is readily verifiable, then assures the result; but in this case invariant measures are readily constructible without reference to any such deep theorem. We may also remark that for 2-step groups, it is quite easy to see that $Q(\hat{P})$ is $T_{1}$; and it is perhaps worth remarking that Haar measure on the cosets of various subgroups invariant by $\mathrm{Ad}^{*} \mathscr{P}$ give examples of positive $\mathrm{Ad}^{*} \mathscr{P}$-invariant measures on $\hat{P}$.

This being the general situation, we now restrict ourselves to some nicer special cases.

Proposition 8. Take $\theta \in Q(\hat{P})$, and suppose any $\mathrm{Ad}^{*} \mathscr{P}$ orbit in $\theta$ is dense. Then $\theta$ supports an $\mathrm{Ad}^{*} \mathscr{P}^{\text {-invariant measure. }}$

Proof. Fix $U$, a compact, clopen subset of $\theta$. Given a compact open subgroup $M \subseteq \mathscr{P}$, let $U(M)$ be the union of the translates of $U$ by $M$, and let $\mu(M)$ be any $\mathrm{Ad}^{*} M$-invariant positive measure on $U(M)$, such that $\mu(M)(U)=1$. Let $F$ be any compact subset of $\theta$. Given $x \in F$, we can, by our assumption on $\theta$, find $g \in \mathscr{P}$ such that $\mathrm{Ad}^{*} g(x) \in U$. Then $\mathrm{Ad}^{*} g^{-1}(U)$ will be a compact neighborhood of $x$. $F$ being compact we may find $g_{1}, \cdots, g_{n}$ such that $F \subseteq \bigcup_{i=1}^{n} \operatorname{Ad}^{*} g_{i}^{-1}(U)$. Then, for any $M$ containing the $g_{i}$, we will have $F \subseteq U(M)$, and $\mu(M)(F) \leqq n$. Thus as $M$ increases to fill $\mathscr{P}$, we may take a $\omega^{*}$-limit of the $\mu(M)$ 's, which will be an $\mathrm{Ad}^{*} \mathscr{P}$-invariant measure on $\theta$.

For closed orbits, we can say more. Not only does an invariant measure exist, but it may be identified with (some multiple of) the canonical trace on the representation space, because the representation with the appropriate kernel is CCR, and $\mathscr{A}(\mathscr{P})$ is mapped to trace class operators.

Before stating the final proposition, we remark that, in direct analogy with Lemma 12 , if $\mathscr{R} \subseteq \mathscr{P}$ is a closed subgroup, and $\psi \in P$ defines a one-dimensional character on $\mathscr{R}$, then $U^{\psi, \mathscr{R}}$ has as kernel in $C^{*}(\mathscr{P})$ the primitive ideal $\alpha(\theta), \theta \in Q(\hat{P})$, if and only if $\psi+R^{\perp} \subseteq \theta$.

Proposition 9. If $\theta$ is a closed $\mathrm{Ad}^{*} \mathscr{P}$ orbit in $\hat{P}$, then an 
irreducible representation with kernel $\alpha(\theta)$ is CCR. In particular, there is only one class of irreducible representation with kernel $\alpha(\theta)$.

Proof. Take $\psi \in \theta$, and suppose $R$ is a polarizing subalgebra for $\psi$, as in Lemma 11. Then $\operatorname{ker} U^{\psi, \mathscr{R}}=\alpha(\theta)$. To verify $U^{\gamma, \mathscr{R}}$ is CCR it suffices to check that for any representation $V$ of any compact open subgroup $M$ of $\mathscr{P}$, the multiplicity of $V$ in $U^{\text {, }}{ }_{1 M}$ is finite. This multiplicity is equal to the sum over double cosets $\mathscr{R} x M$ of the multiplicities with which $\mathrm{Ad}^{*} x(\psi)$ restricted to $x^{-1} \mathscr{R} x \cap M$ is contained in $V$ similarly restricted. Let $\mathscr{A}$ be the isotropy group of $\psi$. Since $\theta$ is closed, it is homeomorphic to $\mathscr{P} / \mathscr{A}$. Since $\mathscr{R} \supseteqq$ $\mathscr{A}$, we may consider the natural map $m: \theta \rightarrow \mathscr{P} / \mathscr{A} \rightarrow \mathscr{P} / \mathscr{R}$.

Let $0 \leqq \log M$ be the $\mathrm{Ad}^{*} M$ orbit of $V$. If $\pi: \hat{P} \rightarrow \widehat{\log M}$, then $\pi^{-1}(0)$ is compact, and so, therefore, is its image under $m$ in $\mathscr{P} / \mathscr{R}$. Since $M$ is open in $\mathscr{P}, m\left(\pi^{-1}(0)\right)$ can consist of only finitely many $M$ orbits. But these orbits correspond to the double cosets $\mathscr{R} x M$ for which the above indicated multiplicity can be nonzero (this is a direct extension of Lemma 12. See also [5]), so the total multiplicity of $V$ in $U^{\psi, \Im}$ is indeed finite.

To conclude, we would like to remark that the methods developed above may be used to extend the results of [8] to the case of a function field over a finite field. Specifically, let $F$ be such a field, of characteristic $p$, and for $k<p$ let $\mathscr{N}$ be any $k$-step nilpotent, unipotent algebraic group over $F$, with lie algebra $N$. Then all localizations of $\mathscr{N}$ are CCR, and the usual orbit picture holds. Suppose $N$ is a vector space over $F$. Let $\mathscr{N}_{A}$ be the adele group of $\mathscr{N}$, and let $\chi$ be a basic character of $F_{A}$ in the sense of [12]. Using $\chi$ we identify $\hat{N}_{A}$ with $N_{A}^{*}$, the algebraic dual. Then the representations of $\mathscr{N}_{A}$ occurring in the natural representation on $L^{2}\left(\mathscr{N}_{A} / \mathscr{N}\right)$ (which is just $U^{1, r}$ ) are exactly those which correspond to orbits in $N_{A}^{*}$ containing points of $N^{*}$. Each representation occurs with multiplicity one. Also, if $C \cong \mathscr{N}_{A}$ is a compact-open subgroup, such that there is a compact open $C^{\prime}$, normalizing $C$, and such that $C^{\prime} \mathscr{N}=\mathscr{N}_{A}$, then the multiplicity with which the identity representation of $C$ occurs in the restriction of $U^{1, r}$ to $C$, may be computed using the Riemann-Roch theorem of [12].

\section{REFERENCES}

1. L. Auslander et al, Flows on homogeneous spaces, Ann. of Math. Studies, no. 53, Princeton Univ. Press, 1963.

2. J. M. G. Fell, The dual spaces of $C^{*}$-algebras, T. A. M.S., 94 (1960), 365-403.

3. M. Hall, The Theory of Groups, The Macmillan Co., 1959.

4. E. Hewitt and K. A. Ross, Abstract Harmonic Analysis, Springer-Verlag, 1963.

5. R. Howe, On representations of discrete, finitely generated, torsion-free, nilpotent 
groups, Pacific J. Math., 73 (1977), 281-305.

6. A. A. Kirillov, Unitary representations of nilpotent Lie groups, Uspekhi. Matem. Nauk., 106 (1962), 57-110.

7. G. W. Mackey, Theory of group representations, University of Chicago, mimeographed notes, 1955.

8. C. C. Moore, Decomposition of unitary representations defined by discrete subgroups of nilpotent groups, Ann. of Math., 82 (1965), 146-182.

9. G. Schiffmann, Distributions centrales de type positif sur un groupe de Lie nilpotent, Bull. Soc. Math. de France, 96 (1968), 347-355.

10. J. P. Serre, Lie Algebras and Lie Groups, W. A. Benjamin, Inc., 1965.

11. - Cohomologie galoisienne, Lecture Notes in Math., 5, Springer-Verlag, 1965.

12. A. Weil, Basic Number Theory, Springer-Verlag, New York, 1967.

Received May 21, 1977.

YALE UNIVERSITY

New Haven, CT 06520 



\title{
PACIFIC JOURNAL OF MATHEMATICS
}

\section{EDITORS}

\author{
RICHARD ARENS (Managing Editor) \\ University of California \\ Los Angeles, CA 90024 \\ Charles W. Curtis \\ University of Oregon \\ Eugene, OR 97403 \\ C. C. MOORE \\ University of California \\ Berkeley, CA 94720
}

\author{
J. DUGUNDJI \\ Department of Mathematics \\ University of Southern California \\ Los Angeles, CA 90007
}

R. Finn and J. Milgram

Stanford University

Stanford, CA 94305

\section{ASSOCIATE EDITORS}

E. F. BeCKenBaCH

B. H. NeumanN

F. WOLF

K. YoSHIDA

\section{SUPPORTING INSTITUTIONS}

\author{
UNIVERSITY OF BRITISH COLUMBIA \\ CALIFORNIA INSTITUTE OF TECHNOLOGY \\ UNIVERSITY OF CALIFORNIA \\ MONTANA STATE UNIVERSITY \\ UNIVERSITY OF NEVADA, RENO \\ NEW MEXICO STATE UNIVERSITY \\ OREGON STATE UNIVERSITY \\ UNIVERSITY OF OREGON
}

\author{
OSAKA UNIVERSITY \\ UNIVERSITY OF SOUTHERN CALIFORNIA \\ STANFORD UNIVERSITY \\ UNIVERSITY OF HAWAII \\ UNIVERSITY OF TOKYO \\ UNIVERSITY OF UTAH \\ WASHINGTON STATE UNIVERSITY \\ UNIVERSITY OF WASHINGTON
}

The Supporting Institutions listed above contribute to the cost of publication of this Journal, but they are not owners or publishers and have no responsibility for its content or policies.

Mathematical papers intended for publication in the Pacific Journal of Mathematics should be in typed form or offset-reproduced, (not dittoed), double spaced with large margins. Please do not use built up fractions in the text of the manuscript. However, you may use them in the displayed equations. Underline Greek letters in red, German in green, and script in blue. The first paragraph or two must be capable of being used separately as a synopsis of the entire paper. Items of the bibliography should not be cited there unless absolutely necessary, in which case they must be identified by author and journal, rather than by item number. Manuscripts, in triplicate, may be sent to any one of the editors. Please classify according to the scheme of Math. Reviews, Index to Vol. 39. All other communications should be addressed to the managing editor, or Elaine Barth, University of California, Los Angeles, California, 90024.

The Pacific Journal of Mathematics expects the author's institution to pay page charges, and reserves the right to delay publication for nonpayment of charges in case of financial emergency.

50 reprints to each author are provided free for each article, only if page charges have been substantially paid. Additional copies may be obtained at cost in multiples of 50 .

The Pacific Journal of Mathematics is issued monthly as of January 1966. Regular subscription rate: $\$ 72.00$ a year (6 Vols., 12 issues). Special rate: $\$ 36.00$ a year to individual members of supporting institutions.

Subscriptions, orders for numbers issued in the last three calendar years, and changes of address should be sent to Pacific Journal of Mathematics, 103 Highland Boulevard, Berkeley, California, 94708. Older back numbers obtainable from Kraus Periodicals Co., Route 100, Millwood, NY 10546.

\footnotetext{
PUBLISHED BY PACIFIC JOURNAL OF MATHEMATICS, A NON-PROFIT CORPORATION

Printed at Kokusai Bunken Insatsusha (International Academic Printing Co., Ltd.). 8-8, 3-chome, Takadanobaba, Shinjuku-ku, Tokyo 160, Japan.
} 


\section{Pacific Journal of Mathematics}

\section{Vol. 73, No. $2 \quad$ April, 1977}

Roger Evans Howe, On representations of discrete, finitely generated, torsion-free, nilpotent groups ........................ 281

Roger Evans Howe, The Fourier transform for nilpotent locally compact

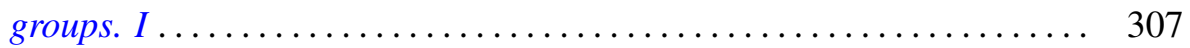

Roger Evans Howe, On a connection between nilpotent groups and

oscillatory integrals associated to singularities............... 329

Roger Evans Howe, Kirillov theory for compact p-adic groups .......... 365

Roger Evans Howe, Topics in harmonic analysis on solvable algebraic

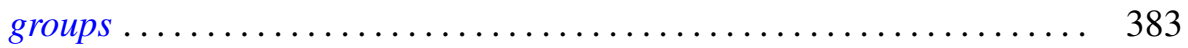

Roger Evans Howe, Tamely ramified supercuspidal representations of

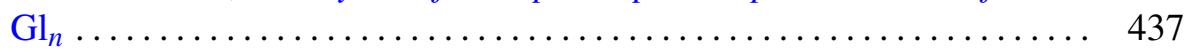

Lawrence Jay Corwin and Roger Evans Howe, Computing characters of tamely ramified $p$-adic division algebras .....................

Roger Evans Howe, Some qualitative results on the representation theory of

$\mathrm{Gl}_{n}$ over a $p$-adic field ............................. 479

Herbert Stanley Bear, Jr., Corrections to: “Ordered Gleason parts”. . . . . . 539

Andreas Blass, Corrections to: "Exact functors and measurable cardinals" .....................................

Robert M. DeVos, Corrections to: "Subsequences and rearrangements of sequences in $\mathrm{FK}$ spaces". 